\title{
El círculo lógico vivencial de la investigación científica como estrategia de enseñanza y modalidad de aprendizaje de metodología de la investigación. Estudio de caso (Ecuador)
}

\author{
Richard, Enrique \\ Universidad Andina Simón Bolívar, Ecuador \\ chelonos@gmail.com
}

\author{
Contreras Zapata, Denise Ilcen \\ Universidad Andina Simón Bolívar, Ecuador \\ dennycz@gmail.com
}

\begin{abstract}
Resumen - El objetivo fue evaluar como estudio de caso, la aplicación vivencial del círculo lógico de la investigación científica como estrategia de enseñanza aprendizaje significativa de metodología de la investigación en la docencia de grado universitaria. Para ello se aplicó la técnica de investigación acción participativa. Los resultados indican que la metodología de la investigación es aprendida y aprehendida en forma efectiva (100\%) si la misma es vivenciada en todos los pasos del círculo lógico desde un rol protagónico. Asimismo, la concientización del rol social, cultural, académico y económico que la misma juega en el desarrollo de un país tuvo una asimilación del $100 \%$. Complementariamente los estudiantes desarrollaron sentido de identidad y pertenencia; aptitudes, destrezas y habilidades de oralidad, pedagogía, capacidad de síntesis, sentido de responsabilidad, ética, respeto académico, autoestima, autocrítica, entre otros.
\end{abstract}

Palabras clave - Estrategia de enseñanza aprendizaje; investigación; estudio de caso;

Abstract - The objective was to evaluate as a case study, the experiential application of the logical circle of scientific research as a teaching strategy of significant learning research methodology in university degree teaching. To this end, the participatory action research technique was applied. The results indicate that the research methodology is effectively learned and apprehended (100\%) if it is experienced in all the

Interconectando Saberes, 2021

ISSN: 2448-8704

(cc) EY-NC-ND steps of the logical circle from a leading role. Likewise, the awareness of the social, cultural, academic and economic role that it plays in the development of a country had an assimilation of $100 \%$. In addition, students developed a sense of identity and belonging; skills, skills and skills of orality, pedagogy, capacity for synthesis, sense of responsibility, ethics, academic respect, selfesteem, self-criticism, among others.

Keywords - Learning teaching strategy; research; case study;

\section{INTRODUCCIÓN}

La UNESCO, finalizando el siglo XX (Reunión de Paris de 1998) delineó los parámetros que marcarían la diferencia entre la Educación Superior Universitaria (ESU) y la Educación Superior No Universitaria (ESNU) a nivel global. En este sentido, estableció que la diferencia entre ambas es la investigación.

La UNESCO indicó que la enseñanza universitaria debe ser de "primera mano". Es decir que la docencia debe estar en manos de las personas que generan o producen el conocimiento que imparten. Fecha de Recepción: 24 de mayo de 2020 Fecha de Aceptación: 10 de diciembre de 2020 Fecha de Publicación: 31 de enero de 2021 
A nivel de posgrado, en países desarrollados es inadmisible que un docente no imparta conocimiento generado por él mismo (Oppenheimer 2010, Richard y Contreras 2014, Crisci y Apodaca 2017, Richard 2017, 2018).

En un contexto nacional es un hecho que un país, una sociedad, jamás podrá salir adelante en sus perspectivas de desarrollo sino genera sus propios conocimientos contextualizados en su realidad y con sensibilidad social $(=$ Independencia académica sensu Richard y Contreras 2014). En otras palabras, la dependencia no sólo incluye el aspecto económico, sino también (o sobre todo) el cultural, científico y consecuentemente académico (Oppenheimer 2010, Richard y Contreras 2014, Richard 2017, 2018).

Para lograr, tanto la independencia, como el desarrollo de un país los docentes deben desarrollar entonces competencias investigativas en los estudiantes. Sin embargo, muchas veces la limitante para ello es la paradoja de las competencias (sensu Richard 2018); es decir "nadie puede desarrollar en el estudiante las competencias que no posee". La falta de tales competencias en muchos docentes ha motivado que los estudiantes al no comprender, ni metodología de la investigación, ni el rol de la investigación en su futuro profesional y el desarrollo de su país, opten por alternativas de graduación que no incluyen tesis o similares. Alternativas que surgen precisamente del problema expuesto (Richard 2006, 2018, Richard y Contreras 2014).
Luego, esta masa mayoritaria de graduados ingresa a posgrados de investigación donde, las falencias formativas en esta área y heredadas del grado se manifiestan y para subsanarlas optan en muchos casos por alternativas no éticas como la compra de la tesis (Barral 2014, Richard 2006, 2017, 2018, Richard y Contreras 2014). Por lo tanto, el primer requisito para alcanzar el objetivo es que el perfil docente incluya competencias efectivas en investigación científica. Pero aún así, no basta tampoco con enseñar metodología de la investigación si no se justifica al estudiantado adecuadamente $y$, en primer lugar, el rol que la misma juega en su futuro profesional como herramienta de resolución de problemas, así como en el desarrollo de un país (Richard 2017, 2018, Richard y Contreras 2014).

En este sentido, ya en 1916 Dewey proponía en un contexto naturalista y sociocrítico la aplicación del método científico en la docencia y si bien desde hace al menos un par de décadas se viene trabajando en distintas formas de aprendizajes basados en investigación (ABI's, sensu Boyer 1998, Travé y Pozuelos 2008, Torres 2012, Peñaherrera et al 2014, inter aliis); las mismas intentan promover la investigación como actividad; pero, fuera del contexto académico y social que implica el círculo lógico de la investigación científica (Richard 1997, 2003, Richard y Contreras 2014).

Además, sin incluir en los contenidos la importancia de la investigación científica en el desarrollo del país y en la profesión. Consecuentemente, el estudiante ve a la investigación como actividad 
aislada o compartimentalizada respecto a su realidad, pero sin vivenciar su rol y responsabilidad social en el cambio de la realidad de un contexto social determinado, local, regional o global.

En función a lo expuesto, el objetivo del presente trabajo es proponer y evaluar por competencias el círculo lógico de la investigación científica como estrategia de enseñanza aprendizaje significativo y vivencial de la metodología de la investigación en la docencia de grado universitaria.

\section{MATERIALES Y MÉTODOS}

El presente estudio se enmarca en el paradigma de investigación naturalista sociocrítico y el método aplicado es el de investigación acción participativa (IAP) (fide Balcazar 2003, Melero 2012). Es decir, los estudiantes son los "directores y actores" vivenciales de su propia obra. La implementación del método se detalla en una guía elaborada "ad hoc" modificada y actualizada del modelo publicado por Richard et al (2004) para la implementación del método.

Dicha guía fue socializada en un grupo de estudios creado en

Facebook (https://www.facebook.com/groups/MICCAU/) para el desarrollo de la asignatura Metodología de la Investigación (Primer semestre, cohorte 2019, $n=$ 41 estudiantes, todos de la generación Z) (sensu Días et al 2015), de la carrera de Ingeniería Ambiental de la Escuela Superior Politécnica Agropecuaria de Manabí, Manuel Félix López (ESPAM MFL) (Calceta, Manabí, Ecuador).
El primer día de clases se realizó una evaluación diagnóstica de conocimientos en torno a los contenidos de la asignatura y aspectos contextuales de la misma. La primera semana de clases se explicó la importancia de la investigación científica en el desarrollo social, tecnológico, cultural y económico con ejemplos ilustrativos a nivel global, regional y local y cómo los logros de tales investigaciones resultan consecuentes, en la escala que fuere de la aplicación del círculo lógico de la investigación científica. (Richard y Contreras 2014)

\section{Figura 1: Círculo lógico de la investigación científica.}

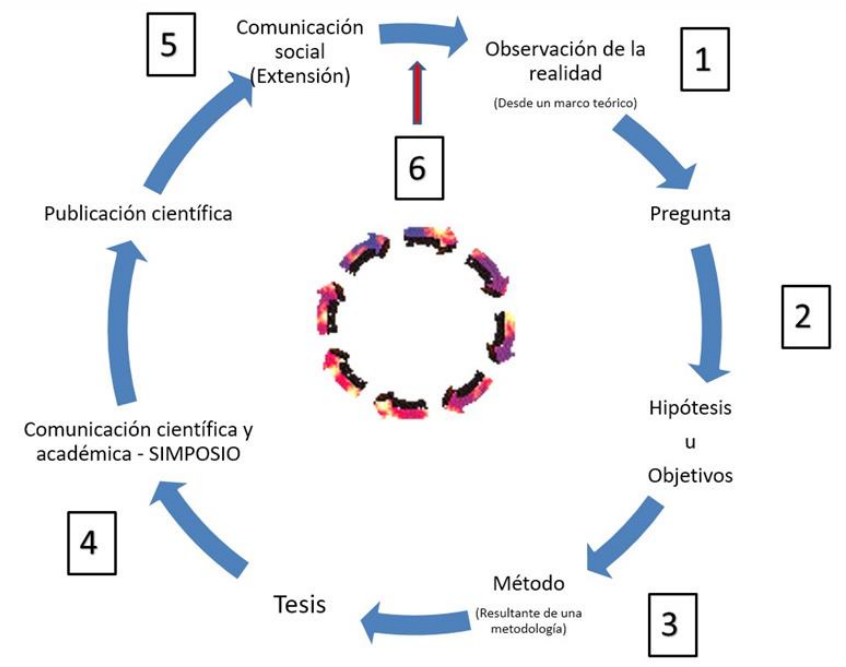

Fuente: Modificado de Richard y Contreras 2014

Descripción del circulo lógico de la investigación científica: 1) Instancia evaluativa del marco teórico y tema de investigación elegido; 2) Evaluación de la pregunta o planteo del problema, la/s hipótesis u objetivo; 3) Evaluación del método y justificación de este; 4) Evaluación final del trabajo de investigación terminado en dos instancias, un presimposio dentro 
de la cátedra (Especialmente para evaluar los resultados, discusión y conclusiones de las investigaciones) y el simposio autogestionado por los estudiantes (La investigación completa); 5) Proceso de comunicación social poscurso de los trabajos de investigación y su potencial impacto social que deriva en el aporte al cambio de realidad, 6) de la cual se partió. Todas las instancias evaluativas incluyen la defensa oral de las mismas y el avance escrito de la investigación. (Modificado de Richard y Contreras 2014)

Tras la explicación de cómo funciona el círculo lógico de la investigación científica, se les propuso a los estudiantes llevarlo a cabo y vivenciarlo completamente a lo largo de la asignatura; desarrollando a lo largo de la misma un trabajo de investigación original. Para ello se planteó (segunda semana de clases), acorde lineamientos de la guía (Richard et al 2004, Richard 2015) lo siguiente (Explicación in extenso en la citada guía):

1. El círculo lógico de la investigación en sus primeras etapas (Elección del tema y fundamentación del mismo, incluido manejo de fuentes bibliográficas), desarrollo del marco teórico, formulación y/o planteo del problema, hipótesis y/u objetivos, métodos y técnicas, resultados y discusión, conclusiones como parte de las evaluaciones parciales de la asignatura. Estas etapas del círculo se evaluaron bajo la modalidad de avance escrito y defensa oral con retroalimentación tanto del docente como de los compañeros (Instancias 1 a 3 Fig. 1). La concreción del trabajo final y su defensa frente a un tribunal externo de especialistas en el marco de un evento (Simposio, instancia 4 Fig. 1) fue voluntario y opcional al examen final escrito de la asignatura. Con ello se buscó que la actividad refleje vocación y motivación y no sea vista como una actividad impuesta por el docente.

2. Los trabajos de investigación se realizaron en forma individual o en grupos de no más de 4 estudiantes. El número máximo sugerido fue tomado en base a evaluaciones de ABI's (Camacho et al 2002) entre otros, en los cuales se demuestra que grupos mayores sufren de una mayor dispersión y menor compromiso con las actividades.

3. Cada grupo eligió un tema de investigación vinculado a la carrera y acorde a un relevamiento previo de antecedentes. La elección libre de temas fue una motivación importante. Cuando los estudiantes investigan un tema de su completo agrado o que responda estrictamente a una línea vocacional propia se maximiza el rendimiento de la investigación (Richard 2017, 2018).

4. A cada grupo se le pidió identificarse con un nombre y un lema que represente e identifique la filosofía de su investigación. Esta actividad estuvo dirigida a promover el sentido de identidad y pertenencia, así como una sana competitividad intergrupal en el desarrollo de la investigación (Camacho et al 2002, Richard et al 2004). Los grupos constituidos tuvieron fueron de carácter permanentes a lo largo del semestre, con lo cual se busca aumentar el afianzamiento y sociabilización de sus miembros en forma horizontal. Esto promovió complementariamente el ejercicio de 
responsabilidad, sentido de identidad y pertenencia, la motivación endógena y el aprendizaje colaborativo/cooperativo, entre otros.

5. Cada grupo eligió un representante para integrar una comisión organizadora de un simposio formal de características propias (Evento Richard fide Camacho et al 2002) que tuvo la responsabilidad durante todo el semestre de organizar un simposio académico formal acorde a la guía suministrada (Cfc. Richard et al 2004, Richard 2015). Esto incluyó entre otros: Elaborar un cronograma de actividades, incluyendo topes para la presentación de los resúmenes y trabajos completos. Búsqueda de financiamiento y su administración (Monitoreada por los docentes de la cátedra como órgano de contralor externo del evento). Diseño de publicidad y difusión de la misma (Impresa y on line) (Ver https://www.facebook.com/events/186924102 680121/?active_tab=discussion y Anexo I). Diseño de una circular con las instrucciones de autores para los resúmenes y trabajos completos. Estas actividades promovieron, entre otros, la capacidad de autogestión y autonomía de los estudiantes, autoestima, sentido de responsabilidad (Camacho et al 2002, Richard et al 2004, Richard 2015).

6. Para la elaboración de los trabajos finales se estableció las características estructurales más universales de un artículo científico: Título, autores, institución, objetivos, materiales y método, recolección de datos, conclusiones, bibliografía, anexos. Todas ellas, condiciones mínimas de forma y fondo exigidas en toda reunión científica (cfc Daciuk 1979; Gastel 1983): El trabajo completo en su versión final fue presentado a la cátedra para su evaluación por parte del tribunal evaluador externo 48 horas antes del evento.

7. De la exposición: Se fijaron las siguientes pautas a fin de evaluar lo aprendido (Aspectos disciplinar y pedagógico): Todas las exposiciones serían orales con el fin de promover y desarrollar aptitudes y destrezas en oralidad, didáctica y pedagogía. Tendrían 15 minutos como máximo y cinco de discusión (Desarrollo del sentido de responsabilidad y capacidad de síntesis). Se puso a disposición de los expositores los recursos pedagógicos necesarios: Proyector de diapositivas y pizarra acrílica. La moderación del evento fue realizada por estudiantes de la cátedra designados por la comisión organizadora y preparados a tal fin con una charla informativa impartida por la cátedra. La evaluación de los trabajos y su defensa fue realizada por un tribunal externo formado por el Director de Investigación de la ESPAM MFL, dos docentes de la carrera afines a la asignatura, una docente de la asignatura, pero de otra carrera y una docente de la asignatura de otra institución y país. La rúbrica de evaluación incluyó tanto los aspectos disciplinares como los pedagógicos (Richard 2015). El evento se realizó con todas las formalidades del caso fuera de aula, en el Auditorium de la ESPAM MFL (Ver Anexo I)

8. Del monitoreo del evento: La cátedra actuó como órgano de control y monitoreo de todas las actividades anteriores. Una encuesta de 
carácter anónimo elaborada exprofeso fue entregada al finalizar el evento a fin de evaluar la respuesta "retorno" de la experiencia por parte de los estudiantes.

9. De la clausura del evento: Para la misma se previó el diseño y la entrega de certificados de exposición, asistencia, participación como organizadores y moderadores. Además, se tuvo en cuenta la entrega de certificados premiando a los mejores trabajos (votados por el tribunal evaluador externo).

10. Otros aspectos: La cátedra se mantuvo abierta a cualquier sugerencia surgida del seno de la comisión organizadora y los estudiantes. El evento en su totalidad fue grabado en vídeo a fin de evaluar luego, con más detalle, los resultados, imperfecciones, falencias $y$ aspectos a mejorar en el mismo.

Finalmente se previó que cada grupo de investigación eligiera un representante para realizar un programa de radio donde se difundan la importancia de las investigaciones realizada a la sociedad y de esa forma cumplir con el cierre del círculo lógico de la investigación científica.

\section{RESULTADOS Y DISCUSIÓN}

De un total de 41 estudiantes, 29 (=70,7\%) voluntariamente aceptaron como modalidad evaluativa final completar el círculo lógico de la investigación científica a través de la realización de un simposio autogestionado con la defensa oral y pública de sus trabajos de investigación involucrando a un tribunal externo imparcial y finalmente difundiendo la importancia de sus conocimientos a la sociedad. El resto de los estudiantes, optaron por la modalidad de examen final escrito, pero participaron en el simposio en calidad de oyentes y apoyando a sus compañeros en diferentes actividades organizativas. La semana previa al simposio la cátedra organizó un pre simposio como último proceso de retroalimentación de los trabajos finales, especialmente la instancia de resultados, discusión y conclusiones, así como las exposiciones orales.

El simposio fue publicitado en las redes sociales de los estudiantes y como evento en Facebook (https://www.facebook.com/events/186924102680

121/). Asimismo, se enviaron invitaciones a todas las carreras de la ESPAM MFL y carreras afines de otras universidades (Anexo I). Se diseñó un poster y un banner para el evento (Anexo I). Dadas las formalidades del evento, el mismo fue realizado en el Auditorium de la institución (Anexo I). El $100 \%$ ( $n=29)$ de los estudiantes que expusieron sus investigaciones en el simposio aprobaron. El promedio de las rúbricas de los cinco evaluadores fue de 91/100 ( $n=29,100 \%$ ), con un mínimo de $82 / 100(n=2,6,9 \%)$ y un máximo de $100 / 100(n=4$, $13,8 \%$ ). Todos los participantes recibieron certificados con valor curricular acorde a su grado de participación en el mismo (Anexo I). Las categorías fueron la siguientes: Expositor, participante, autor, coautor, comisión organizadora, maestro de ceremonia, protocolo, difusión.

Las encuestas realizadas a la totalidad de los estudiantes ( $n=41,100 \%)$ arrojaron los siguientes resultados (Partim): Antes del cursar "Metodología 
de la Investigación" sólo el 12,2 \% (n=5) indicó conocer la utilidad que poseía la investigación para la ciencia, el desarrollo social, cultural y/o para cambiar la realidad de la sociedad. Luego de vivenciar el círculo lógico de la investigación en el semestre, dicho porcentual fue del $100 \%(n=41)$. A pesar del desconocimiento inicial de la utilidad de la investigación científica, la percepción de la mayoría de los estudiantes al inicio del semestre respecto a la importancia de la investigación en la carrera fue mayoritaria $(90,2 \%$ grado 5 y $9,8 \%$ grado 4 en escala de Likert de 1 al $5, n=41$ ).

Probablemente esto último se deba a la percepción generalizada previa de que en la universidad la investigación es una de las funciones sustantivas más importantes. Dicha percepción o bien la traen de su formación anterior o con mayor certeza de las charlas motivacionales que reciben en la ESPAM MFL durante el curso prefacultativo. El porcentual con relación a la importancia de la investigación en la carrera, luego del simposio fue del $100 \%$ para el grado 5 en la escala de Likert.

A la pregunta de "En cuantas asignaturas les hicieron defender oralmente y acorde a los lineamientos de un simposio un trabajo de investigación", el 100 \% respondió que sólo en "Metodología de la Investigación". Sin embargo, esta respuesta no tiene valor representativo dado que se trataba de estudiantes del primer semestre y donde solo cursan cinco materias.
Dos días después del simposio (Grabado) y cierre de la materia, se realizó una reunión y conversatorio en los que participó el $100 \%$ de los estudiantes que expusieron sus investigaciones $(n=29)$. Los mismos $(100 \%)$ atestiguaron $y$ demostraron tener un dominio de la lógica de la investigación científica conforme a lo impartido en clases.

Acorde a sus testimonios, el haber vivenciado y protagonizado la investigación a lo largo de un semestre en sus diferentes etapas y sobre un tema de elección propia resultó muy motivador como estrategia de enseñanza aprendizaje.

Por otro lado, trabajar en grupos permanentes a lo largo de un semestre identificados con un logo y lema identificatorio, les promovió el sentido de identidad y pertenencia hacia el grupo motivando aún más el trabajo de investigación (93,1 \% para el grado 5 en la escala de Likert, 6,9\% para el grado 3 y $4, n=29)$.

El desarrollo del sentido de identidad y pertenencia es importante para el funcionamiento y el bienestar sicológico y social de las personas, ya que conduce a emociones positivas como el gozo, la calma, el entusiasmo y la felicidad además de propiciar resultados productivos (Brea 2014).

El sentido de identidad puede tener una gran amplitud de significados, interpretaciones e implicaciones (Cfc. Bordieu 1982, Navarrete 2014) pero en lo que a esta investigación concierne, 
consideramos que implica "identificarse" con un tema de investigación por un lado y con un grupo social por el otro. En este último caso, con su ideología, su filosofía, su forma de trabajo y esto es fundamental como factor sinérgico de trabajo.

Trabajar, investigar plenamente identificado con el tema y con las personas del grupo de trabajo es una motivación fundamental para llevar a buen puerto la misma. Acorde con Hopenhayn et al. (2011) "El Sentido de Pertenencia a una comunidad es uno de los factores que permite que las personas puedan ejercer sus opciones de vida de modo real. Es en comunidad con los demás como las personas obtienen reconocimiento, definen sus proyectos de vida y, gracias a la relación con ellos, pueden llevarlos a cabo" (p.154).

Desde el punto de vista psicológico pertenencia ha sido definida como el "sentido de participación personal en un sistema social en donde las personas se sienten a sí mismos como una parte indispensable e integral del sistema" (Anant 1966, in Hagerty et al., 1992 p.173). Consecuentemente, la identificación con un tema y grupo y el sentido de pertenencia a este último son factores motivacionales sustantivos para que el proceso de investigación acción participativa se lleve a cabo.

La apropiación de la investigación y de los pasos del círculo lógico de la investigación científica depende en gran medida de la estimulación de estos factores por parte del docente. Luego del simposio, el $100 \%$ de los participantes $(n=29)$ admitió que la práctica de exponer los avances de las investigaciones a lo largo del semestre y prepararse posteriormente para exponer públicamente el trabajo terminado en un tiempo estipulado, frente a un tribunal evaluador externo contribuyó notablemente al desarrollo de autocrítica y aptitudes, destrezas y habilidades de oralidad, pedagogía, capacidad de síntesis, así como de lectoescritura (93,1\% para el grado 5 en la escala de Likert, 6,9\% para el grado 3 y 4, $n=29$ ). Esto es de suma importancia teniendo en cuenta que la universidad se enfrenta actualmente con el problema de las nuevas adicciones, las tecnológicas (Carbonell et al 2008, Ruiz et al 2010, Gutiérrez 2018, inter aliis).

Los estudiantes actuales (Generación Z fide Días et al 2015) llegan al aula y no pueden desprenderse de sus celulares, a pesar de los reglamentos universitarios que limitan y condicionan su uso y de las políticas de aula que los restringen aún más. Esta dependencia y adicción deriva en alteraciones emocionales, disociación con la realidad y comportamientos antisociales, entre otros (Sánchez et al 2008, Carrillo et al 2017, Gutiérrez 2018). Por otro lado, también motiva a que las nuevas generaciones no lean textos, ni escriban en su lenguaje. En lugar de ello utilizan para comunicarse los sociolectos propios de la era del celular y contextualizados en cada región (Días et al 2015, Fernández 2019).

Luego, al ingreso a la universidad los estudiantes deben leer artículos científicos, libros de textos con un lenguaje notablemente enriquecido respecto al sociolecto del celular y presentan serias 
dificultades de lectura, comprensión y posterior escritura en informes. Hecho que como hemos podido evidenciar en al menos los últimos 10 años en diferentes cursos y universidades de Latinoamérica. Frente a ello, los estudiantes optan por el consabido "copiar y pegar" (Fontúrbel 2004, Richard 2006), escribir párrafos enteros en mayúscula, omitir puntuación y otras "patologías" ortográficas y gramaticales.

En este sentido, los estudiantes atestiguaron que la metodología aplicada los motivó a leer artículos científicos. Lo cual derivó, de acuerdo a sus opiniones, en una mayor comprensión lectora, menor dispersión de la atención, aumento exponencial del vocabulario (sic) y mayor facilidad para escribir. Por otro lado, el trabajo grupal de equipo estimuló las actividades de socialización "cara a cara" (93,1 \% para el grado 5 en la escala de Likert, 6,9 \% para el grado 2 y 4, $n=29$ ) en lugar de hacerlo a través del celular, donde notablemente y al menos para las tareas de investigación, el sociolecto aplicado dejó de tener eficacia. Esta actividad entonces contribuyó notablemente a fomentar la socialización y desarrollo de habilidades interpersonales de los estudiantes, una seria deficiencia de la generación $Z$ (Días et al 2015). Las exposiciones de los avances en forma aleatoria (Solo exponía uno o dos miembros de cada grupo sorteados por el docente antes de la defensa) motivó que todos los miembros se prepararan y ensayaran la defensa en cada instancia evaluativa con la retroalimentación permanente del grupo en su preparación y luego del curso completo en la exposición.
Con ello se generó una práctica permanente de las habilidades de oralidad, pedagogía, capacidad de síntesis, uso de lenguaje, lectura y escritura. El cambio cualitativo en estos aspectos quedó evidenciado al comparar los primeros trabajos presentados y expuestos oralmente con los de final de semestre.

Complementariamente, el hecho de pasar un semestre completo revisando bibliografía les ayudó notablemente a comprender y entender la importancia del respeto académico hacia los autores que trabajaron en líneas de investigación similares; la importancia de conocerlos a través de sus obras y de citarlos adecuadamente. En otras palabras, la ética académica (82,7\% grado 5 y 17,2 $\%$ grados 2, 3 y 4 en la escala de Likert de 1 al 5, $\mathrm{n}=29$ ). De acuerdo a sus testimonios, hacer una investigación les permitió valorar el trabajo de investigación ajeno, algo que antes normalmente no hacían. Esto es particularmente importante en tiempos actuales en los cuales, los estudiantes universitarios y demasiados docentes también, consideran que lo que está en la red no tiene autor (Richard 2006, 2017, 2018).

El plagio en muchos lugares de Latinoamérica se ha institucionalizado (Gómez et al 2016) y la venta de tesis, tanto de grado como de posgrado, se realiza públicamente en los clasificados de periódicos o aún peor con publicidad en la calle y en el interior de las propias universidades (Gómez et al 2016, Richard 2017, 2018). 
Curiosamente, al preguntarles por el uso del celular admitieron que en realidad consideraban que a lo largo del semestre usaron mucho más el celular respecto a lo que normalmente hacían. Pero que esto se debía a que ahora destinaban más tiempo a la lectura de artículos en formato pdf $(89,6 \%$ grado 5 y 10,3\% grado 2, 3 y 4 en la escala de Likert de 1 al 5, n=29). Es decir, si bien el uso del celular en su percepción se incrementó, también en su percepción, comenzaron a darle un uso más "productivo" (Sic).

Asumir y aprender que la ciencia avanza rompiendo paradigmas, equivocándose, cometiendo errores ayudó mucho a ver la crítica externa como algo positivo y digno de capitalizar. Consecuentemente también ayudó al desarrollo de la autocrítica (79,3\% grado 5 en la escala de Likert, $20,7 \%$ grado 1 a $4, n=29$ ), proceso sinergizado por la práctica cotidiana del debate constructivo (Intragrupal e intergrupal) y las retroalimentaciones durante las defensas de avance de los trabajos. Esto es especialmente importante tomando en cuenta que justamente una de las características de la generación $Z$ es la hipersensibilidad a la crítica y falta de autocrítica (Días et al 2015, Rincón 2017). Finalmente, el hecho de crear comisiones y organizar el simposio en forma totalmente autónoma y autogestionada promovió en ellos (86,2 \% grado 5 en la escala de Likert, 13,8 \% grado 3 y $4, n=29$ ) el sentido de responsabilidad y autoestima. En todos los casos tanto la autoestima, como la seguridad en sí mismos, estuvo sinergizada por el avance y progreso del trabajo de investigación, sumado al hecho de realizar diseños experimentales propios y obtener resultados $(93,1$
\% para el grado 5 en la escala de Likert, 6,9\% para el grado 4). En este sentido, tal vez el indicador más relevante de autoestima y seguridad en sí mismo haya sido el hecho de que los estudiantes invitaran a sus padres al simposio donde expondrían (Con valor de examen final) y, sobre todo, los hicieran venir de sus ciudades de origen, teniendo en cuenta que todos participantes del simposio $(n=29$, 100\%) no son de Calceta.

La UNESCO, en la reunión de Paris de 1999 propuso como objetivo universal de la educación superior "Formar seres humanos íntegros". Objetivo adaptado, modificado, pero de una u otra forma incorporado en los estatutos universitarios de Latinoamérica. Sin embargo, a la fecha, se sigue descuidando el hecho de que "formar" no es lo mismo que "informar" (cfc. Richard 2017). Formar implica necesariamente incorporar los valores como los aquí citados, en forma transversal e integral, al tiempo de compartirles a los estudiantes los conocimientos disciplinarios propios de la asignatura (Richard 2004, 2017, 2018).

Formar implica necesariamente darles las herramientas y orientación que les permita a los estudiantes encontrar el camino de su felicidad haciendo lo que les gusta. Enseñarles a disfrutar el proceso de enseñanza aprendizaje. Es por ello que la presente experiencia, al incorporarlos en un rol protagónico y responsable en su formación, dentro de una estrategia de investigación acción participativa, ha demostrado ser eficaz y eficiente cumpliendo el objetivo planteado por la UNESCO (1999). Sin embargo, no es menos cierto que el 
método necesita como requisito un perfil docente pertinente y competente. Como lo indicara Richard (2004, 2006, 2017, 2018), el docente como parte de su perfil debe poseer dominio del tema, característica que le permite ser una persona segura de sí misma. Seguridad que se traduce en el discente en credibilidad y esta última, en el motor de los procesos formativos. Nadie cambia, si no cree en la persona con la que interactúa (Richard y Contreras 2012).

En las diferentes formas de aprendizajes basados en investigación practicadas actualmente se intenta promover la investigación como actividad, pero fuera (Total o parcialmente) del contexto académico que implica el círculo lógico de la investigación científica (Richard 1997, 2003, Richard y Contreras 2014). Consecuentemente, el estudiante ve a la investigación como actividad aislada o compartimentalizada respecto a su realidad académica y profesional. Consecuentemente, sin vivenciar su rol y responsabilidad en el cambio de la realidad de un contexto social determinado, local, regional o global. En otros casos, se proponen con carácter de ABI's, investigaciones bibliográficas en corto tiempo para ser expuestas en aula (cfc. Castro 2017). Pero esta estrategia no da el tiempo para el afianzamiento y/o apropiación del método científico, ni para el desarrollo de aptitudes, destrezas, habilidades y valores aquí citados e involucrados.
El método aquí propuesto entonces plantea el recorrido semestral o anual vivencial del círculo lógico de la investigación científica (Richard y Contreras 2014) sobre la base de un único tema, abordado en forma individual o grupal y donde el grupo es en sí mismo un equipo de investigación.

Con evaluaciones expositivas parciales que representan el constructo de un artículo científico y finalmente la organización autogestionada de un simposio de características especiales (Anexo I y II) donde los estudiantes cierran el componente investigativo defendiendo oral y públicamente sus artículos frente a un tribunal externo que precautela la evaluación imparcial.

En todo este proceso, que tiene algunos componentes del aprendizaje por problemas y de indagación (Cfc. Rubio et al 2013, González 2014, Peralta 2014), el estudiante es protagonista permanente y responsable tanto de la organización del simposio, su formalidad académica, como de los cronogramas de avance de la investigación a lo largo del semestre. Todo ello en el contexto de la investigación acción participativa.

En todos los casos, el desarrollo y estimulación de los factores mencionados (Identidad, pertenencia, oralidad, pedagogía, capacidad de síntesis, lectoescritura, autocrítica, autoestima, responsabilidad, ética académica, entre otros) son elementos motivacionales sustantivos necesarios para que el proceso de investigación acción participativa se lleve a cabo y exista un empoderamiento del mismo. Como se indicó previamente la apropiación de la investigación y de 
los pasos del círculo lógico de la investigación científica dependerá en gran medida de la estimulación de estos factores por parte del docente.

Finalmente, luego del conversatorio mantenido con los estudiantes, cada grupo eligió un representante como vocero del equipo de investigación para realizar un programa de radio en vivo (Richard 2020). Este último paso es crucial en la comprensión real de la función sustantiva de vinculación con la comunidad o extensión. Función que epistemológicamente, debe emerger o se consecuente de la investigación. Los estudiantes tuvieron el desafío de explicar cómo sus investigaciones pueden mejorar de una $u$ otra forma diferentes aspectos de la sociedad. Para ello también se plantearon el desafío de transducir (Sensu Richard 2004, 2006) el lenguaje científico utilizado en sus artículos, en un lenguaje coloquial más accesible. Esta actividad y la interacción con el público que llamaba a la radio en vivo, les permitió comprender, no solo la importancia social de la investigación sino también vivenciar en forma protagónica cómo dichas investigaciones pueden aportar un granito de arena al cambio de la realidad de donde partieron sus investigaciones; cerrando con ello vivencialmente el círculo lógico de la investigación científica.

\section{CONCLUSIONES}

El método utilizado en el presente trabajo demostró una eficacia y eficiencia absoluta (100\%) en el aprendizaje y apropiación vivencial del círculo lógico de la investigación científica. Los estudiantes finalizando el curso son conscientes y comprenden la importancia de la investigación científica en el desarrollo social, cultural, económico y académico local, regional y global de la humanidad. Comprenden al círculo lógico de la investigación como una parte importante de su futura vida y ejercicio profesional. Perciben perfectamente el rol de la investigación como factor de transformación y cambio de la realidad (100\%).

Complementariamente el método contribuye sustancialmente al objetivo universal de la educación (Formar seres humanos íntegros, UNESCO 1999) con el desarrollo y afianzamiento de aptitudes, destrezas, habilidades y valores (Sentido de identidad y pertenencia, oralidad, pedagogía, capacidad de síntesis, lectoescritura, autocrítica, autoestima, responsabilidad, respeto, ética académica, convivencia, autogestión, autonomía, entre otros)

\section{AGRADECIMIENTOS}

Los autores expresan su mayor agradecimiento al Director de Investigación de la ESPAM MFL, Dr. Ángel Guzmán Cedeño; a la Dra. Rosa Victoria Gonzáles Zambrano docente de Metodología de la Investigación de la carrera de Medicina Veterinaria de la ESPAM MFL y a los Ing. Carlos Delgado Villafuerte y Carlos Villafuerte Vélez, docentes de la carrera de Ingeniería de Ingeniería Ambiental de la ESPAM MFL por su participación en carácter de tribunal evaluador del Simposio. Finalmente, a la M. Sc. Zaira Velásquez Cedeño, Coordinadora de Comunicación de la ESPAM MFL por la cobertura del evento. 


\section{REFERENCIAS}

Balcázar, F. E. (2003) Investigación acción participativa (IAP): Aspectos conceptuales y dificultades de implementación.

Fundamentos en Humanidades, 4 (7): 59-77

Barral Z. R. (2014) Educar o adoctrinar: análisis crítico del currículo de la ley $n^{\circ} 070$ "Avelino Siñani-Elizardo Pérez" y otros temas de ecología, geopolítica, cultura y lectura. Ediciones Brecha. 344p. La Paz. Bolivia Bourdieu, Pierre (1982). "La identidad como representación”, en G. Giménez, (comp.). La teoría y el análisis de la cultura, México: SEP/Universidad de Guadalajara/Consejo Mexicano de Ciencias Sociales, A. C.

Boyer Commission Report (1998). The Boyer Commission on Educating Undergraduates in the Research University, Reinventing Undergraduate Education: A Blueprint for America's Research Universities.

Recuperado el 8 de febrero de 2020 de : http://www.niu.edu/engagedlearning/researc h/pdfs/Boyer Report.pdf

Brea, L. M. (2014). Factores determinantes del sentido de pertenencia de los estudiantes de Arquitectura de la Pontificia Universidad Católica Madre y Maestra, Campus Santo Tomás de Aquino. Recuperado el 8 de marzo de 2020 de:

https://www.tdx.cat/bitstream/handle/10803/2 84952/TLMBA.pdf?sequence $=1$

Camacho, A; A. Zurita, A. Miranda, V. Mariaca, M.E. Delgado, R. Silva, J. Romero y G. Rojas. (2002). Análisis de la metodología de evaluación del Diplomado en Gestión del Proceso de Enseñanza Aprendizaje planteada por el Dr. Enrique Richard (Evento Richard). En: Richard, E.; J. Murillo \& D. Contreras Z. 2003. El Evento Richard como estrategia de enseñanza y modalidad de aprendizaje para transversalizar componentes actitudinales de investigación, entre otros. Fundamentos teórico prácticos (4ta Ed., Versión 2003). Guía docente de la Maestría en Gestión y Evaluación del Proceso de Enseñanza Aprendizaje Universitario y Metodología de la Investigación, gestión y Comunicación Científica y Académica. UMSA, Fac. Ing., Serie Apuntes $n^{0}$ IV. La Paz, Bolivia.
Carrillo, G., B. L. Zuñiga, B. Toscano, N. Aguas G. \& J. C. Díaz M. (2017) Los dispositivos móviles e Internet y su uso en el aula: ¿Un factor distractor en el proceso de aprendizaje? Un estudio de caso. Rev. CONAIC 4 (3): $40-51$

Castro, I. (2017) La exposición como estrategia de aprendizaje y evaluación en el aula Apuntes básicos para docentes. Editorial Razón y Palabra. Facultad de Comunicación- Universidad de Los Hemisferios. Quito. Ecuador. $311 \mathrm{p}$.

Crisci J. y J. Apodaca (2017). Los rankings globales de universidades y su función disciplinaria. Rev. Museo La Plata 2 (2): 1218

Daciuk, J. (1979) Guía para la preparación y publicación de las contribuciones científicotécnicas en el campo de la Ornitología y de las Ciencias Naturales en general. Hornero 012 (01): 023-028.

Dewey, J. (1916). Democracia y educación. Buenos Aires: Losada.

Dias, C., N. P. Caro \& E. J. Gauna (2015). Cambio en las estrategias de enseñanza-aprendizaje para la nueva Generación $Z$ o de los "nativos digitales". Virtual Educa 14: 164-175

Fernández, C. (2019). La evaluación de sociolectos: creencias y prejuicios lingüísticos. Revista electrónica de estudios filológicos, Tonos digital 36: 1-25

Fontúrbel, F. (2004). ¿Qué sería de los bolivianos sin el copy-paste? En: Richard, E. (Ed.). Cátedra de Educación Ambiental, Maestría en Recursos Naturales y Gestión Ambiental, Escuela Militar de Ingeniería. CD-ROM Interactivo. Escuela Militar de Ingeniería y Ed. Multimedia Theratos, La Paz, 41p.

Gastel, B. (1983) Presenting science to the public. ISI Press. $146 \mathrm{p}$.

Gómez- Espinosa, M., Francisco, V., \& MorenoGer, P. (2016). El impacto del diseño de actividades en el plagio de Internet en educación superior. Comunicar, 48, 39-48. Doi: https://doi.org/10.3916/C48-2016-04

González, M. A. (2014). Estrategias didácticas sobre el aprendizaje basado en la indagación. Actas I Congreso y 16 Simposio de Estrategias Didácticas en el Aula. Universidad Nacional Autónoma de México. Recuperado el 10 de marzo de 2020: https://www.eventos.cch.unam.mx/congreso simposioestrategias/memorias/\#menu 
Gutiérrez, J. (2018). El uso problemático del teléfono móvil: desde el abuso a su consideración como adicción comportamental. Tesis Doctoral. Universidad Complutense de Madrid, Facultad de Psicología, Departamento de Psicobiología. Recuperado el 10 de marzo de 2020: https://eprints.ucm.es/46732/1/T39651.pdf

Hagerty, B., Lynch-Sauer, J., Patusky, K., Bouwsema, M. y Collier, P. (1992). Sense of Belonging: A Vital Mental Health Concept. Archives of Psychiatric Nursing, 4(3), pp. 172- 177. Recuperado el 10 de marzo de 2020 de http://deepblue.lib.umich.edu/bitstream/handl e/2027.42/29998/0000365.pdf

Hopenhayn, M. y Sojo, A. (Ed.). (2011). Sentido de Pertenencia en sociedades fragmentadas: América Latina en una perspectiva global. Buenos Aires: Siglo XXI.

Melero, N. (2012). El paradigma crítico y los aportes de la investigación acción participativa en la transformación de la realidad social: un análisis desde las ciencias sociales. Cuestiones Pedagógicas, 21: 339-355

Navarrete-Cazales, Z. (2015). ¿Otra vez la identidad?: Un concepto necesario pero imposible. Revista mexicana de investigación educativa, 20 (65): 461-479. Recuperado en 10 de marzo de 2020, de http://www.scielo.org.mx/scielo.php?script=s ci arttext\&pid=S1405$66662015000200007 \&$ Ing=es\&tlng=es.

Oppenheimer, A. (2010). ¡Basta de historias! La obsesión latinoamericana con el pasado y las 12 claves para el futuro. Editorial Debate. México. 272 p.

Peñaherrera, M., Chiluiza, K. y Ortiz, A. (2014). Inclusión del Aprendizaje Basado en Investigación $(\mathrm{ABI})$ como práctica pedagógica en el diseño de programas de postgrados en Ecuador. Elaboración de una propuesta. Journal for Educators, Teachers and Trainers, 5 (2): $204-220$.

Peralta, D. M. (2014) Investigar para aprender estrategia didáctica para modelar con matemáticas. Actas I Congreso y 16 Simposio de Estrategias Didácticas en el Aula. Universidad Nacional Autónoma de México. Recuperado el 10 de marzo de 2020:

https://www.eventos.cch.unam.mx/congreso simposioestrategias/memorias/\#menu
Richard, E. (2004). Universidad, docencia, política y sociedad en Bolivia. Reflexiones en torno al perfil docente investigador universitario ideal para el tercer milenio, en Bolivia. Pp 3 - 41. En: Barral Zegarra, R. (Ed.). 2004. Educadoras nuevas, educadores nuevos. Editorial Ayni Ruway. La Paz, Bolivia. 144 p. Richard, E. (2006). Investigación científica y soberanía nacional: Reflexiones en torno a la problemática de la investigación científica y la soberanía nacional en Bolivia. Pp. 99 148. En: Barral Zegarra, R. y E. Richard (Eds). Educación y constituyente: Autonomías y soberanía. 148 p. Ed. Ayni Ruway. La Paz, Bolivia. ISBN 99905-0-0405.

Richard, E. (Ed), (2014). Guía de Actividades y Trabajos Prácticos, Cátedra de Metodología de la Investigación Científica (Grado y Postgrado). En: E. Richard (ed.) Metodología de la Investigación Científica y Taller de Tesis (Grado y Postgrado), Materiales de Estudio y Guía de Actividades Prácticas. Universidad Mayor de San Andrés. CD ROM Interactivo + VCD. Ed. E. R. Multimedia. La Paz, Bolivia. DL 4-4-230607, ISBN 978-99905-841-8-9.

Richard, E. (2017). Praxis profesional en la Universidad del siglo XXI. Ensayo. Pp 3 23. En: Actas VI Evento Internacional "La Universidad en el Siglo XXI". Escuela Superior Politécnica Agropecuaria de Manabí Manuel Félix López. Manabí, Ecuador. Ecuador.

Richard, E. (2018). La investigación científica y la praxis profesional en la universidad del siglo XXI: Panorama de Bolivia y Latinoamérica. Ensayo. Pp: 69-85. En: L. Vargas Espinoza, J. Tinto Arandes, M. Florez Guzman, E. Herrera de Alvarado, E. Gea Izquierdo (Comp.). Avances e Innovación en la Administración y Gerencia en Salud. CIDE Centro de Investigación y Desarrollo Ecuador. ISBN: 978-9942-759-59-7

Richard, E. (DrEnriqueRichard) (2020, febrero 11) I Simposio de Estudiantes de Metodología de la Investigación. Recuperado de https://www.youtube.com/watch?v=nyeiSp32nw\&t=16s 
Richard, E.; J. Murillo P. \& D. I. Contreras Zapata (2004). El Evento Richard como estrategia de enseñanza y modalidad de aprendizaje para transversalizar componentes actitudinales de investigación, entre otros. Fundamentos teórico prácticos (4ta Ed., Versión 2004). Guía docente de la Maestría en Gestión y Evaluación del Proceso de Enseñanza Aprendizaje Universitario y Metodología de la Investigación, Gestión y Comunicación Científica y Académica. UMSA, Fac. Ing., Serie Apuntes $n^{0}$ IV. La Paz, Bolivia. ISBN 99905-0-384-2

Richard, E. \& D. I. Contreras Z. (2012). Educación Ambiental para el Tercer Milenio: Un enfoque epistemológico, pedagógico, legítimo y legal. Instituto de Investigaciones, Convenio Andrés Bello. 418 p. La Paz. Bolivia.

Richard, E. y D. I. Contreras Z. (2014). El rol de la investigación universitaria en la descolonización e independencia académica: Lo que no se publica no existe... UMSA Revista Tribuna Docente 2014 (2): 3 - 5. La Paz, Bolivia.

Rincón, F. (2017) La libertad de expresión en la época de la generación hiper. Ola política 12 de octubre. Recuperado el 8 de marzo de 2020 de http://olapolitica.com/analisis/lalibertad-de-expresion-en-la-epoca-de-lageneracion-hiper/

Rubio, B. A., A. Rubio \& M. Serrano V. (2013). La investigación como una estrategia didáctica para un aprendizaje significativo. Actas I Congreso y 16 Simposio de Estrategias Didácticas en el Aula. Universidad Nacional Autónoma de México. Recuperado el 10 de marzo de 2020:

https://www.eventos.cch.unam.mx/congreso simposioestrategias/memorias/\#menu

Sánchez C., X, M. Beranuy, M. Castellana, A. Chamarro y U. Oberst (2008). La adicción a Internet y al móvil: ¿moda o trastorno? ADICCIONES, 20 (2): 149-160

Torres, A. (2012). Aprendizaje basado en la investigación. Técnicas didácticas. Tecnológico de Monterrey. Recuperado el 20 de enero de 2014 en http://rodin.uca.es:8081/xmlui/bitstream/hand le/10498/15117/713 Penaherrera.pdf?sequence $=7$
Travé, G. y Pozuelos, F. J. (2008). Enseñar economía mediante estrategias de investigación escolar. Estudio de caso sobre las concepciones y prácticas del profesorado. Enseñanza de las Ciencias Sociales, 7, 109-120.

UNESCO, (1999). Conferencia mundial sobre Educación Superior, 1998: La educación superior en el siglo XXI. CD ROM Interactivo. Ed. Unesco. Paris. 


\section{ANEXO I:}

Difusión del evento en la institución, redes sociales, e imágenes del evento
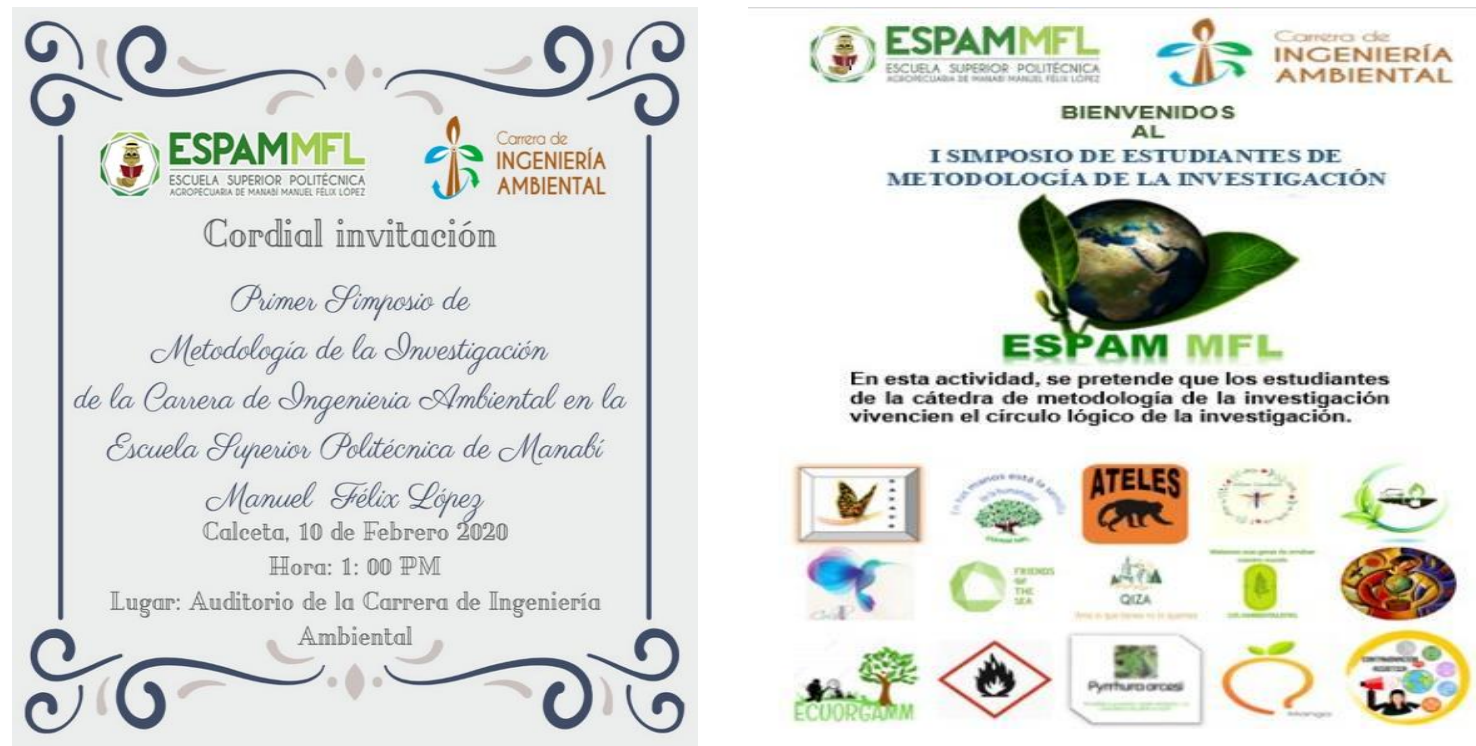

Figs. 2 y 3: Izquierda: Invitación formal distribuida en papel y digital. Derecha poster formal del evento

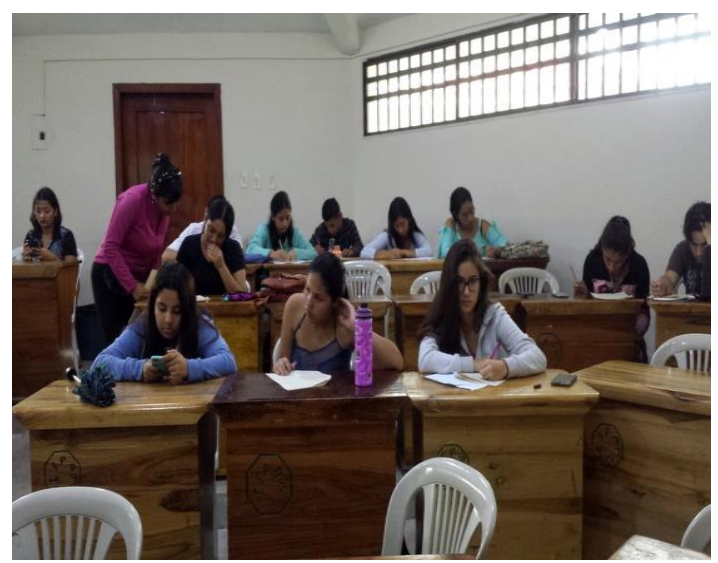

Fig. 4: Durante la realización de la encuesta inicial. Foto: E. Richard

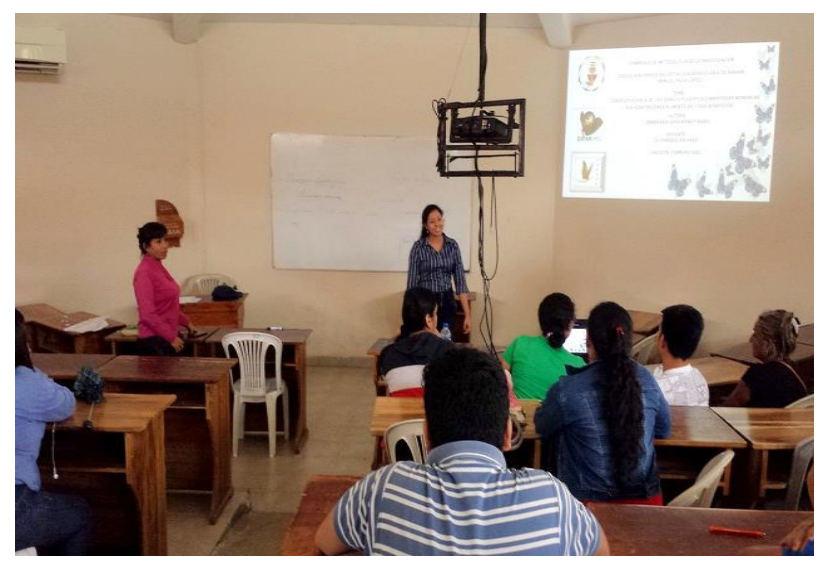

Fig. 5: Retroalimentación de cátedra en el presimposio.

Foto: E. Richard 


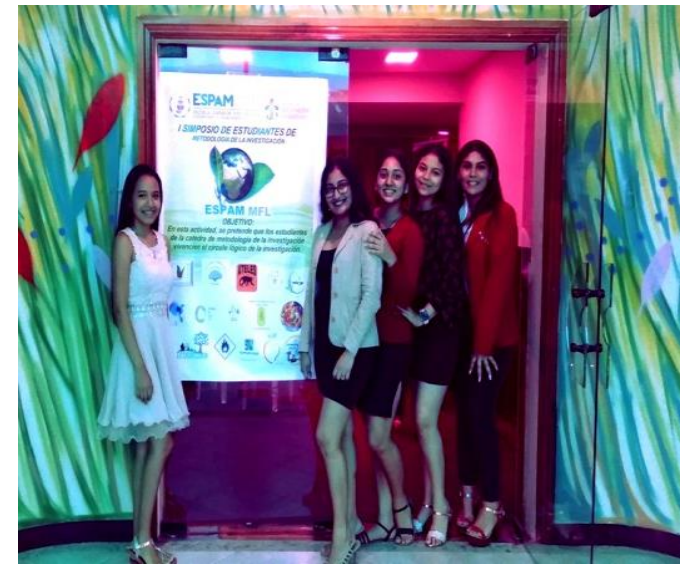

Fig. 6: Momentos previos al inicio del evento en el Auditorium de la ESPAM MFL. Foto. Denise Contreras.

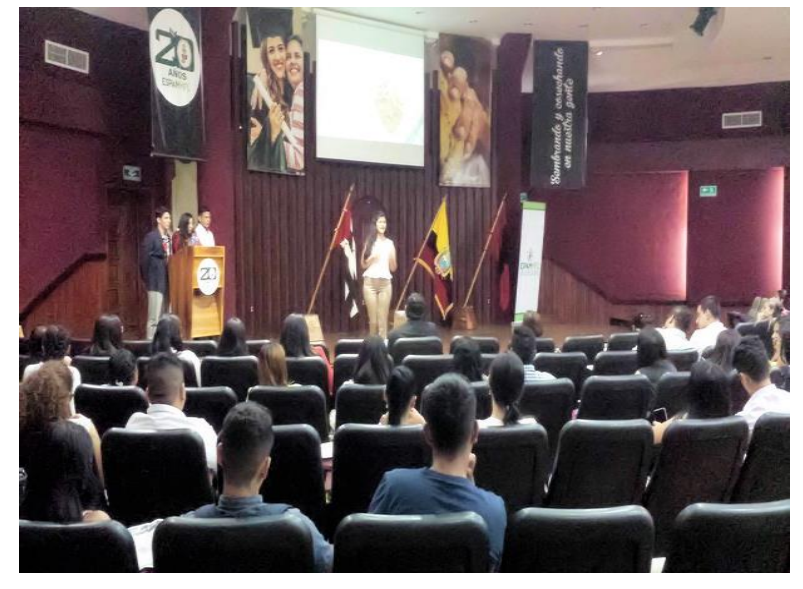

Fig. 7: Exposición de los estudiantes en el auditórium. Foto: E. Richard.

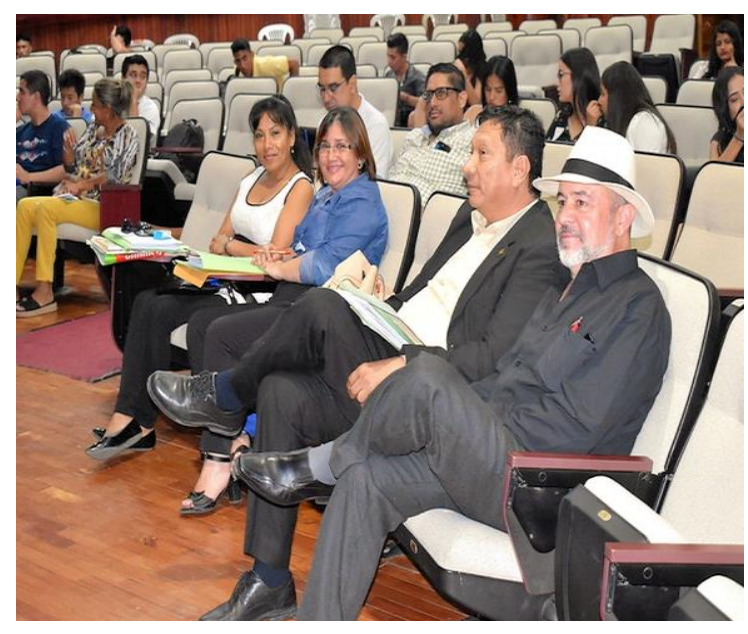

Fig. 8: Docentes y comité evaluador externo. Foto: ESPAM MFL

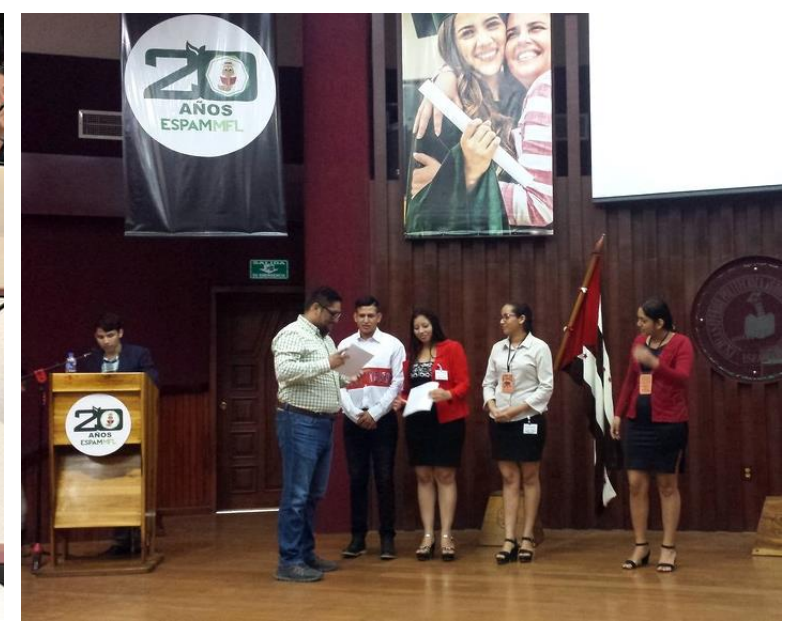

Fig. 9: Ing. Carlos Delgado V. entregando los certificados al cierre del evento. Foto: E. Richard 

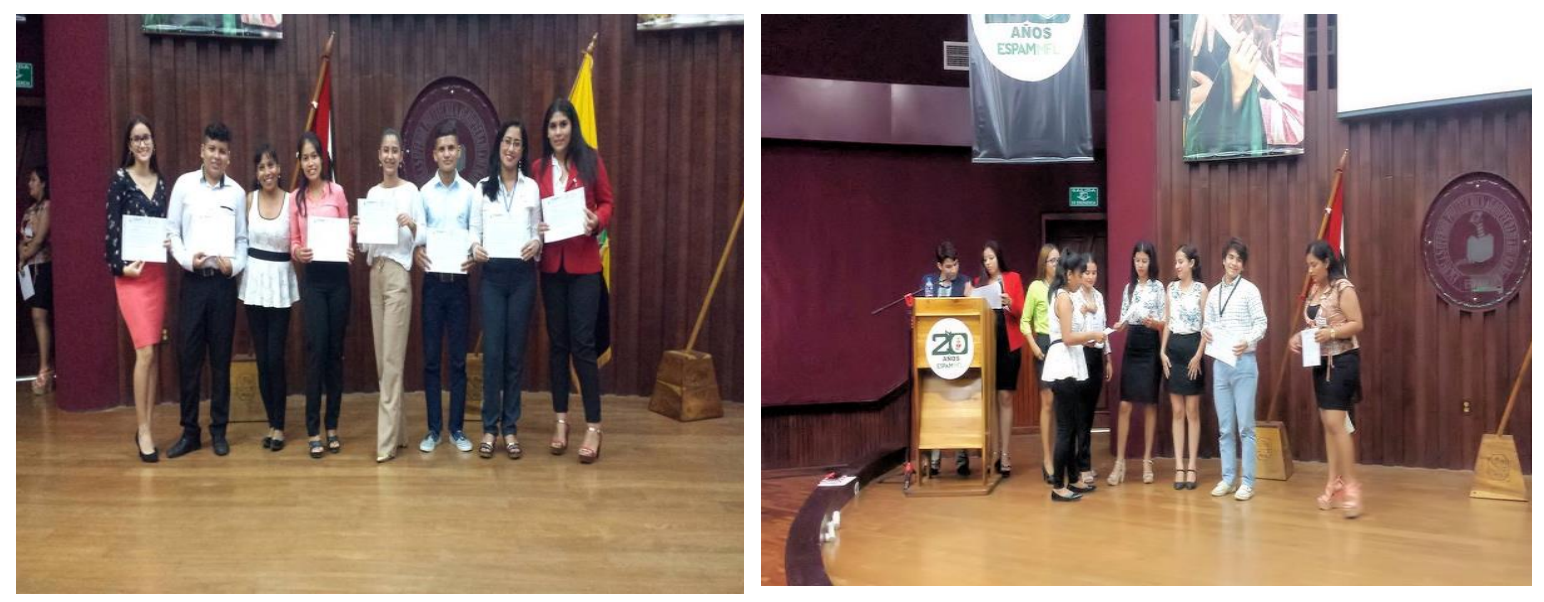

Fig. 10 y 11: Dra. Denise Contreras entregando certificados al cierre del evento. Fotos: E. Richard

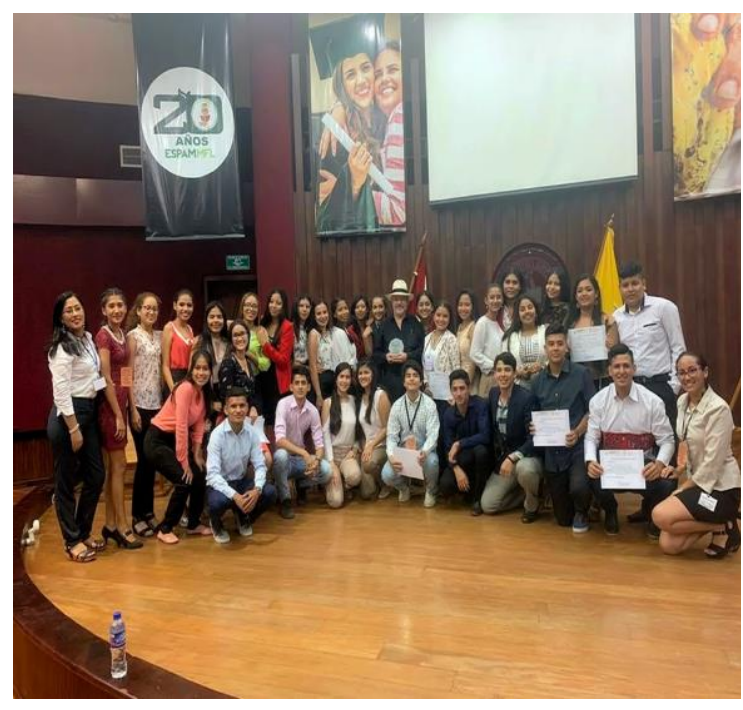

Fig. 12: Cierre del evento. Foto: D. Contreras

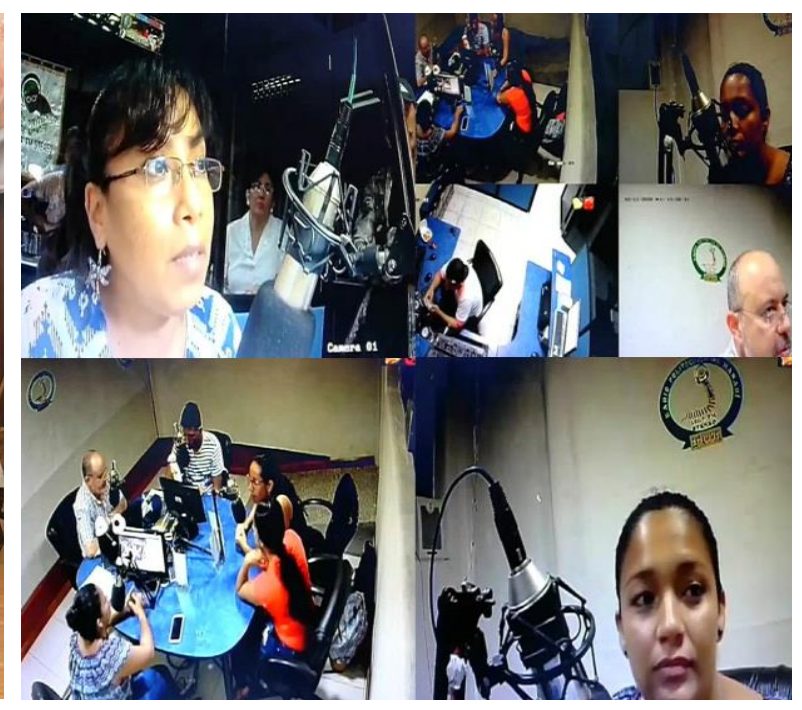

Fig. 13: Programa de radio realizado con los jóvenes investigadores. Foto: ESPAM MFL 


\section{ANEXO II:}

Cuadro comparativo de las principales diferencias conceptuales entre un simposio pedagógico tradicional $\left(^{*}\right)$ y el simposio estudiantil aquí propuesto

$\left({ }^{\star}\right)$ Emergente como sistema de evaluación en estrategias de enseñanza de exposición tradicional, aprendizaje por problemas, aprendizaje por indagación, aprendizajes basados en investigación y en exposición acorde a varios autores (Peñaherrera, M., Chiluiza, K. y Ortiz, A, Rubio et al 2013, González 2014, Peralta 2014, Castro 2017, inter aliis)

\section{Simposio estudiantil (Este trabajo)}

Se basa en una dinámica de grupo permanente con afianzamiento y sociabilización de sus miembros en forma horizontal. Los grupos son estables y de larga duración (Meses o años) con lo que se promueve en trabajo en equipos interdisciplinarios, bajo presión en forma evaluable (Permeabilidad).

Se realiza y de hecho se motiva su realización en diferentes ámbitos extra áulicos.

El tema es único y por tanto investigado y madurado bajo el esquema de una

investigación formal en el marco del ciclo lógico de la investigación científica a lo largo del semestre o año

Roles funcionales formales, variados y con rotación de roles. Las transversales

actitudinales se reflejan en todas las instancias del evento: moderación, organización, investigación, autorías, etc.

Existen elementos cohesionantes de grupo: Nombre, logo y lema (Sentido de identidad y pertenencia). La organización del evento, la filosofía interna de trabajo, etc.

Permite promover e incentivar las actitudes, destrezas, habilidades de: oralidad, pedagogía, capacidad de síntesis, lectoescritura, autocrítica, autoestima, responsabilidad, respeto, ética académica, conviviencia, autogestión, autonomía, entre otros

Se transversaliza una parte importante de la vida profesional futura en el ejercicio y práctica cotidiana del estudiante

La evaluación es permanente a lo largo de la asignatura, objetiva e imparcial (Evaluadores externos) en el final

Se transversaliza y ejercita el concepto de par académico con la evaluación externa

\section{Simposio pedagógico tradicional $\left(^{*}\right)$}

Los grupos son de constitución espontánea y coyuntural. No evaluables en su dinámica interna. La duración es efímera (una clase o un tema) y el grupo también.

Se realiza in situ, en el aula, únicamente. Es coyuntural a la actividad áulica.

La investigación se realiza con insumos proporcionados por el docente en el momento. El ejercicio suele ser informal y generalmente no metódico

Roles limitados a exposición y elaboración, improvisados e informales. Coyunturales

No hay otro elemento cohesionante que la coyuntura de trabajar juntos en ese momento, por ese solo tema, para esa asignatura...
No
No

No

No 


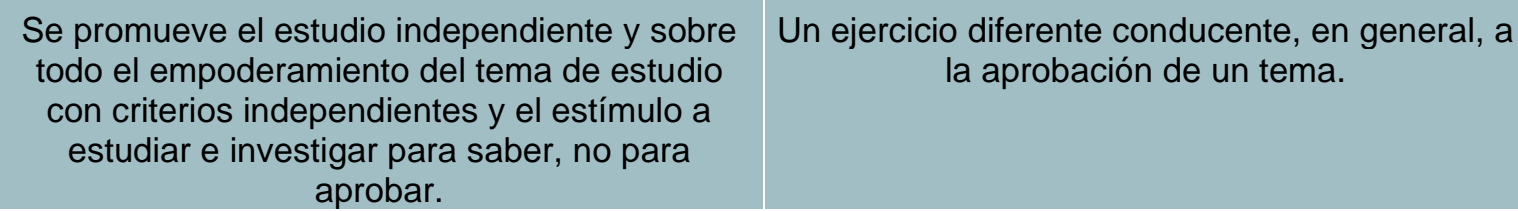

Permite un seguimiento personalizado de los estudiantes discriminando lo actitudinal de lo disciplinar.

Evaluación coyuntural en el momento de la exposición y presentación del trabajo

\begin{tabular}{c|c} 
Permite desarrollar políticas de motivación & No \\
académica y personal tales como cuadros de \\
honor, fechas de onomásticos, etc.
\end{tabular}

Desarrolla la autoestima individual y grupal a través de los logros emergentes del saber hacer y su producto: La investigación, la gestión y la publicación...

Transversaliza los parámetros del paradigma productivo.

Promueve el cambio cualicuantitativo actitudinal y disciplinar tangible, evaluable en el estudiante

Se realiza una vivencia formal y auténtica del ciclo del investigador científico, no un simulacro.

Evento formal realizado fuera de las aulas No

La práctica tiene valor curricular (Presentación de un trabajo en un evento científico, etc.) con certificación oficial

No

Coyuntural

No o se hace un simulacro.

Práctica áulica normal

Está asociado a tareas de vinculación y

No

extensión (Difusión social del conocimiento

científico generado) 